An apparatus for the measurement of low-pressure deviations from the ideal gas laws in the temperaturerange of $50-200^{\circ} \mathrm{C}$. was shown by the Organic Group; the apparatus is suitable for the study of those organic compounds which have a vapour pressure of at least $200 \mathrm{~mm}$. in that temperaturerange. Also shown was an apparatus, still under construction, which has been designed for the precise determination of the latent heats of vaporization of organic liquids, and for the measurement of the specific heats (at constant pressure) of organic vapours; the working temperature-range for this apparatus is likewise $50-200^{\circ} \mathrm{C}$.

Of the new developments shown in organic intermediates, the following were of particular interest : a novel method of carboxylation based on the use of sodium ethyl carbonate; the carboxylation of chrysene and pyridine through their sodio-derivatives; the synthesis of a variety of polymeric materials containing the hydrazino group. Particularly noteworthy was the use of $\mathrm{N}$-amino oxazolid-2-one as a source of $\mathrm{N}$-amino ethyleneimine and its polymer, polyethylene hydrazine; the syn. thesis of arsenic-conteining polymers from arsenic pentoxide and epoxides, and the trimerization of aromatic isocyanates in solution in various epoxides by a trace of pyridine.

There is at present considerable interest in the use of electrodialysis for the removal of salt from sea water, or from brackish waters, which are unfit for drinking or irrigation purposes. Successful application of this process depends on the efficiency of the ion-exchange membranes in multi-compartment electrolytic cells. In the High Polymers Group, methods for the preparation of ion-exchange membranes are being investigated, and a study is being made of their electrochemical properties. Membranes prepared by incorporating particles of an ion-exchange resin in an inert binder may contain void spaces which can lead to inferior electrochemical behaviour. Emphasis has therefore been placed on the preparation of homogeneous ion-exchange membranes, and promising cation- and anion-exchange membranes of this type have been prepared.

The possibilities of paper strip chromatography for the analysis of polymers are also being studied in an attempt to find a simple routine procedure for evaluating molecular weight distributions. A synthetic polypeptide (polysarcosine dimethylamide) served as a convenient model polymer since it can be prepared with very sharp molecular weight distributions. Mixed samples of polysarcosines of molecular weights up to 12,000 have been successfully fractionated on paper strips. The best results were obtained by gradient elution, that is, pumping a good solvent steadily into a reservoir of poor solvent from which ascending development took place. In most cases a polymer labelled with carbon-14 was used for assessing the chromatograms.

The Microbiology Group made a feature of the economic and industrial importance of bacteria taking part in the natural sulphur cycle. Various exhibits explained the activities of these bacteria in polluting aviation fuel oils and coal gas, their function in the disintegration of concrete and in the corrosion of buried metals, and the microbial formation of sulphur in Nature. Of special interest was a process, devised at Teddington and now being developed by the London County Council, for producing sulphur from sewage sludge and gypsum by a semi-continuous fermentation. Continuous techniques, which may well constitute the next important technological advance in the fermentation industries, were also illustrated, and included a novel adaptation of the conventional bubble-cap distillation column. The National Collection of Industrial Bacteria displayed its comprehensive range of assay organisms, which are extensively used by industrial and other research workers for estimating various amino-acids, vitamins and antibiotics.

\section{INTERNATIONAL COLLOQUIUM ON INTERFERENCE SPECTROSCOPY}

$T$ HIS colloquium, organized by the Centre National de la Recherche Scientifique, was held at Bellevue, near Paris, during September 9-13.

Interferometric spectroscopy might appear a rather narrow field for an international conference extending over five days. In fact, the organizer, Prof. P. Jacquinot, proved to be wise in imposing a restriction which went as far as excluding diffraction gratings, when used by themselves, as too conventional : there was ample variety, not only of details but also of fundamentally now and interesting approaches. The number of talks and the size of the audience could be kept in reasonable bounds, allowing worth-while discussions to develop. The proceedings of this most stimulating conference are to be published in the Journal de Physique and should well represent the main lines of the recent advances in this field. Methods, rather than results, formed the subject of the conference.

Interferometers have a fundamental advantage compared with gratings or prisms, in a way which has been most clearly expressed by Jacquinot in the form of a coefficient of merit. In the latter instruments the path difference depends strongly on the angle, a fact which necessitates the use of a slit. This restriction of area in the plane of the slit, or of angle in the collimated beam, entails loss of light gatherod. In contrast to this, interferometers use interference 'in depth', with path differences depending on the angle only to the second power, as shown in the axial symmetry of interference patterns. By imaging an extended source in the plane of an interference pattern rather than on a narrow slit or slitimage, one can gather more light from it.

Full advantage of this can be taken in recording methods where a circular stop is placed in the centre of the fringe pattern, followed by a detector, such as a photo-multiplier. While the path difference is varied at a uniform rate, for example, by variation of the air pressure, the intensity is recorded. This method has lately been increasingly used, and various ways of changing the path difference were described at the conference (R. Chabbal and M. Soulet, J. H. Jaffe, J. Gobert, J. Roig, R. Dupeyrat); besides an alternative method in which the intensity is recorded directly against the air pressure, without a time base (H. G. Kuhn and J. L. Lucas-Tooth).

The quantum efficiency of photocells is generally higher than that of photographic plates, but while the latter collect energy of all wave-lengths simultaneously during the whole time of the exposure, in the recording technique different wave-lengths act 
successively, each only for a fraction of the total time of recording. This waste of energy can be avoided by methods which are grouped together by such names as 'Fourier transform method' or 'multichannel spectroscopy'. Their principle goes back to Michelson's interferometric analysis by 'visibility curves'. We imagine the intensity in the centre of the fringe system, produced by a Michelson or other two-beam interferometer, to be recorded while the path difference is changed at a uniform rate, for example by translational motion of one of the reflectors. If the incident light is monochromatic, a pure sine curve will be recorded, and for any arbitrary spectral distribution the record obtrined will be a Fourier transform of the spectrum. In principle, and with certain restrictions which need not be mentioned here, this record can be transformed back into the original spectrum by numerical calculation, or by the use of computers. In actual fact, the primary spectrum has to be limited to a small spectral range, and certain information on the spectrum usually has to be assumed beforehand. Instead of the absolute wave-numbers, differences against a reference wavenumber are often measured.

The difficulty of making the motion exactly uniform, and the small path difference obtainable in practice, limit the method to low rosolving powers and mainly to the infra-red. In this spectral range, on the other hand, the gain in light-gathering power which this method achieves is most valuable: because of the photon noise in the receiver, the resolving power obtainable is often limited by the intensity. This applies particularly to astrophysical measurements for which the method offers considerable promise. A number of speakers discussed the principle of the method and described actual devices putting it into practice (P. Fellgett, G. A. Vanasse, J. and P. Connes, E. Ingelstam, P. Jacquinot, H. A. Gebbie, L. Merz).

A most unorthodox system was described by $\mathbf{P}$. Connes, who uses two plane gratings to form an interferometer; they take the place of the reflectors in a Michelson interferometer and are slowly and synchronously rotated. At any given moment, light of that wave-length for which the incident and reflected wave-fronts are parallel is modulated by a longitudinal displacement of one of the gratings-in practice achieved by a to- and fro- rotation of the compensator. The device acts as a grating used with direct recording, but the absence of a slit ensures the gain in intensity characteristic of interferometers.

P. Connes also described the construction and the first successful experiments with his ingenious 'spherical Fabry-Perot interferometer'. Between the partially reflecting, spherical surfaces the wave suffers four successive reflexions before it interferes with the direct wave, as compared with the two reflexions in the plane Fabry-Perot. The path difference is independent of the angle not only to the first power of small angles as in the ordinary etalon, but even to the second. This allows a greater solid angle to be used in recording, with a resulting gain in intensity. The instrument offers considerable advantages when very large path differences are required, and the adjustment is extremely easy. The spacing cannot, of course, be varied, and this will restrict the use of the instrument to special purposes.

The use of multiple dielectric films as coatings for étalon plates were treated by F. A. Jenkins, P. Giacomo, A. Steudel, R. Lennuier, J. Ring, R.
Dupeyrat and F. Abelès. Two recent advances in this field will make dielectric coatings much more attractive to spectroscopists than they have been hitherto : the width of the reflexion band can now be made large enough to cover an appreciable portion of the visible spectrum, and films can be made for use in the ultra-violet down to $2500 \mathrm{~A}$.

Photographic interferometry, as a well-established method, very naturally played a smaller part at the conference. Any idea that it has now been superseded by recording methods and had become obsolete would be quite mistaken. Its great power of integrating and of simultaneously recording a large range of the spectrum will ensure its retention, and it is often preferable when wave-numbers rather than intensities are to be measured accurately. Contributions on precision measurements with a double étalon (D. A. Jackson) and on solar interferometry (P. J. Treanor) served as useful reminders of these facts.

Metrological applications, designs of special interferometers and various other topics were discussed. These have to be omitted from this report not for their lack of importance but for reasons of brevity and because they cannot easily be grouped together.

H. G. KUHN

\section{INTERNATIONAL UNION OF CRYSTALLOGRAPHY \\ CONGRESS IN MONTREAL}

$T$

HE International Union of Crystallography held its fourth General Assembly and International Congress in Montreal, during July 10-17. About six hundred crystallographers from nineteen countries attended, accompanied by about one hundred and fifty wives (or husbands) and children. The Congress was followed by two symposia held during July 17-19. A full programme of social events and excursions of scenic and technical interest was arranged, and firstclass facilities were provided by McGill University. During the evening spent at the Université de Montréal the retiring president, R. W. G. Wyckoff, spoke on "Electron Microscope Studies of Macromolecules", with beautiful photographic illustrations.

In the report on the meetings of the International Union of Crystallography held in Madrid in $1956^{1}$, it was commented that the arrangement of several simultaneous sessions was unsatisfactory, and that a number of small meetings, more restricted in scope and with a single subject only claiming attention at any one time, would be more valuable. This difficulty of fragmentation had been felt by the organizers of the Montreal Congress, and a number of general lectures were arranged on several mornings at a time when there was no competition from other meetings. These formed a valuable feature of the Congress, and provided a painless method for specialists in other branches to become acquainted with the present state of erystallography of proteins (D. Hodgkin), erystal chemistry (G. S. Źdanov), interatomic distances and molecular configurations (D. G. Jenkins, J. S. Taylor and L. E. Sutton, presented by L. E. Sutton), clay minerals (G. W. Brindley), and imperfect structures (P. B. Hirsch). In most of these fields there was steady progress but no striking new developments to report. Prof. G. S. Ždanov presented some unusual 